OPEN ACCESS

Edited by:

Zhulong Chan,

Wuhan Botanic Garden (CAS), China

Reviewed by:

Liezhao Liu,

Southwest University, China

Yi Ding,

Wuhan University, China

*Correspondence:

Binying Fu

fubinying@caas.cn

Zhikang Li

lizhikang@caas.cn

tThese authors have contributed equally to this work.

Specialty section:

This article was submitted to Crop Science and Horticulture,

a section of the journal

Frontiers in Plant Science

Received: 26 August 2016 Accepted: 24 October 2016 Published: 08 November 2016

Citation:

Wang W, Qin Q, Sun F, Wang Y,

$X u$ D, Li Z and Fu B (2016)

Genome-Wide Differences

in DNA Methylation Changes in Two

Contrasting Rice Genotypes

in Response to Drought Conditions.

Front. Plant Sci. 7:1675.

doi: 10.3389/fpls.2016.01675

\section{Genome-Wide Differences in DNA Methylation Changes in Two Contrasting Rice Genotypes in Response to Drought Conditions}

\author{
Wensheng Wang ${ }^{1 \dagger}$, Qiao Qin ${ }^{1 \dagger}$, Fan Sun ${ }^{1}$, Yinxiao Wang ${ }^{1}$, Dandan $X u^{1,2}$, Zhikang Li, ${ }^{1,3 *}$ \\ and Binying Fu', ${ }^{1,3}$
}

\begin{abstract}
${ }^{1}$ Institute of Crop Sciences/National Key Facility for Crop Gene Resources and Genetic Improvement, Chinese Academy of Agricultural Sciences, Beijing, China, ${ }^{2}$ College of Agronomy, Anhui Agricultural University, Hefei, China, ${ }^{3}$ Shenzhen Institute for Innovative Breeding, Chinese Academy of Agricultural Sciences, Shenzhen, China
\end{abstract}

Differences in drought stress tolerance within diverse rice genotypes have been attributed to genetic diversity and epigenetic alterations. DNA methylation is an important epigenetic modification that influences diverse biological processes, but its effects on rice drought stress tolerance are poorly understood. In this study, methylated DNA immunoprecipitation sequencing and an Affymetrix GeneChip rice genome array were used to profile the DNA methylation patterns and transcriptomes of the droughttolerant introgression line DK151 and its drought-sensitive recurrent parent IR64 under drought and control conditions. The introgression of donor genomic DNA induced genome-wide DNA methylation changes in DK151 plants. A total of 1190 differentially methylated regions (DMRs) were detected between the two genotypes under normal growth conditions, and the DMR-associated genes in DK151 plants were mainly related to stress response, programmed cell death, and nutrient reservoir activity, which are implicated to constitutive drought stress tolerance. A comparison of the DNA methylation changes in the two genotypes under drought conditions indicated that DK151 plants have a more stable methylome, with only 92 drought-induced DMRs, than IR64 plants with 506 DMRs. Gene ontology analyses of the DMR-associated genes in drought-stressed plants revealed that changes to the DNA methylation status of genotype-specific genes are associated with the epigenetic regulation of drought stress responses. Transcriptome analysis further helped to identify a set of 12 and 23 DMR-associated genes that were differentially expressed in DK151 and IR64, respectively, under drought stress compared with respective controls. Correlation analysis indicated that DNA methylation has various effects on gene expression, implying that it affects gene expression directly or indirectly through diverse regulatory pathways. Our results indicate that drought-induced alterations to DNA methylation may influence an epigenetic mechanism that regulates the expression of unique genes responsible for drought stress tolerance.

Keywords: DNA methylation, rice, drought stress, epigenetics, MeDIP-seq 


\section{INTRODUCTION}

Drought is the most serious environmental stress limiting crop growth, development, and yield (Farooq et al., 2009). Rice is sensitive to drought stress, and the effects of drought on rice plants vary among genotypes and developmental stages (Datta et al., 1988; Degenkolbe et al., 2009). The genes responsible for the mechanisms underlying rice drought tolerance have been studied for decades. Drought tolerance is a complex trait involving several genetic pathways (Todaka et al., 2015). However, little is known about the role of epigenetic processes during the molecular regulation of rice drought tolerance.

DNA methylation is an epigenetic modification that is important for plant growth and development as well as responses to environmental stresses (Bender, 2004; Meyer, 2015). As one of the earliest detected epigenetic modifications, DNA methylation has been observed in many organisms. The methylation of eukaryotic DNA frequently occurs at the 5 position of cytosine, yielding 5-methylcytosine. Under normal growth conditions, the proportion of cytosines that are methylated in plants is $20-30 \%$ (Finnegan et al., 1998). There is much evidence indicating that changes to DNA methylation considerably affect the ability of plants to respond to environmental stresses (Boyko and Kovalchuk, 2008). Saltresponsive genes are differentially methylated under salt stress conditions, indicating that DNA methylation influences plant responses to environmental stress (Wang M. et al., 2014). Furthermore, altered DNA methylation in response to salt stress is organ- and genotype-specific, and is not directly associated with salt tolerance (Wang et al., 2011b; Karan et al., 2012). Drought stress also induces genome-wide changes to DNA methylation, leading to altered gene expression levels (Wang et al., 2011a; Tang et al., 2014). These observations suggest that DNA methylation influences abiotic stress responses and adaptations.

DNA methylation was first analyzed by high-performance liquid chromatography to assess global DNA methylation changes (Cai and Chinnappa, 1999). However, this technique did not allow the determination of the methylation status at individual gene loci. Methylation-sensitive amplified polymorphism (MSAP) is a modified version of the amplified fragment length polymorphism technique, and was developed to assess the status of cytosine methylation in GGCC genomic fragments (Xiong et al., 1999). However, this method can detect only a few methylated sequences because of the limitations of the isoschizomers used. New sequencing-based technologies to analyze genome-wide DNA methylation, including bisulfite sequencing and methylated DNA immunoprecipitation sequencing (MeDIP-seq), are now commonly used (Li et al., 2010; Taiwo et al., 2012). Bisulfite sequencing was recently used to profile diverse methylomes among cultivated and wild rice lines (Li et al., 2012), analyze DNA methylation patterns in different rice genotypes (Garg et al., 2015) and investigate the DNA methylation changes in pesticide-treated rice plants (Lu et al., 2016). The MeDIP-seq platform has been applied to characterize DNA methylation status during rice seed development (Xing et al., 2015) and fertility transitions of the photoperiod- and thermo-sensitive male sterile line (Hu et al., 2015). However, little is known about the effects of genome-wide cytosine methylation on drought responses in rice plants.

In a previous study, we assessed DNA methylation changes in a drought-tolerant rice line (DK151) and its droughtsensitive recurrent parent (IR64) using the MSAP technique. Many genome site-specific methylation differences were detected in both genotypes under drought stress conditions. Droughtinduced DNA demethylation/methylation patterns in rice may be important for adaptation to drought conditions (Wang et al., 2011a). However, most of the DNA methylation of non-CCGG genomic sites was not detected because of the limitations of the MSAP technique. In the current study, to explore genomewide changes to cytosine methylation in rice lines DK151 and IR64 under drought stress conditions, we used MeDIP-seq to characterize differentially methylated regions (DMRs). We also investigated the effects of DMRs on gene expression using microarrays. The results of this study may help to clarify the epigenetic mechanisms that regulate drought stress tolerance in rice plants.

\section{MATERIALS AND METHODS}

\section{Rice Materials and Growth Conditions}

According to our previous study, DK151 showed significantly improved tolerance to drought over IR64 (Wang et al., 2011a). Further, DK151 showed significantly better performance than IR64 under drought stress conditions (Supplementary Figure S1). Seeds of the drought-tolerant DK151 rice line and its drought sensitive-recurrent parent IR64 were surface-sterilized and germinated at $37^{\circ} \mathrm{C}$ in distilled water for 2 days. The germinated seeds were then transferred to a seedling nursery. Rice plants at the four-leaf stage were transplanted into plastic pots (height: $30 \mathrm{~cm}$; diameter: $35 \mathrm{~cm}$ ) filled with sterilized field soil. Seedlings were grown in a greenhouse at $29 / 22^{\circ} \mathrm{C}$ day/night temperatures at the Institute of Crop Sciences of the Chinese Academy of Agricultural Sciences (Beijing, China). Two healthy seedlings from each genotype were planted in one pot (equidistant from each other). Six replicates (three for MeDIP-seq and three for microarray analysis) were prepared for the drought stress and control conditions.

Drought stress was simulated by withholding water at the tillering stage as previously described by Huang et al. (2014). Well-watered plants were used as controls. The three uppermost leaves were harvested from each sample 3 days after initiating the drought treatment. All samples were immediately frozen in liquid nitrogen and stored at $-80^{\circ} \mathrm{C}$.

\section{Genomic DNA Extraction and Methylated DNA Immunoprecipitation Sequencing}

Genomic DNA was extracted from drought-stressed and control DK151 and IR64 plants using the AxyPrep Multisource Genomic DNA Miniprep Kit (Axygen Biosciences). Each sample consisted of three biological replicates. Libraries prepared using non-immunoprecipitated "input" DNA from three biological 
replicates were sequenced as controls. The MeDIP-seq DNA libraries were prepared according to the protocol described by Li et al. (2010). Briefly, $5 \mu \mathrm{g}$ DNA from each sample was sonicated to produce DNA fragments (100-500 bp). Libraries were then constructed using the Paired-End DNA Sample Prep Kit (Illumina, San Diego, CA, USA). Adapterligated DNA was immunoprecipitated using a monoclonal antimethylcytidine antibody. The specificity of the enrichment was confirmed by quantitative reverse transcription polymerase chain reaction (PCR) using the SYBR Green Master Mix (Applied Biosystems). The enriched methylated fragments and input DNA were purified with DNA Clean \& Concentrator5 columns (Zymo). The purified DNA was then used as the template for adapter-mediated PCR. Amplicon quality and quantity were evaluated using the 2100 Analyzer DNA 1000 chips (Agilent). Ultra-high-throughput 50PE sequencing was conducted using the Illumina HiSeq 2000 system. Raw sequencing data were processed by the Illumina base-calling pipeline.

\section{Methylated DNA Immunoprecipitation Sequencing Data Analysis}

Sequencing adapters were removed and low-quality bases (quality $<20$ ) were trimmed from the $5^{\prime}$ and $3^{\prime}$ ends of reads using an in-house Perl script. The obtained clean reads were then mapped to the rice reference genome (IRGSP-1.0/MSU7) using the default parameters of the BWA program (version 0.7.12). The mapped high-quality reads were used for genome-wide distribution analyses (e.g., distribution among chromosomes and in diverse components of the rice genome).

Model-based analysis of MeDIP-seq data (Zhang et al., 2008) was used to detect highly methylated regions (peaks), and the peak locations were summarized with a Perl script. The Picard program $^{1}$ converted mapping results to the BAM format. We used the Bioconductor package MEDIPS (Lienhard et al., 2014) to identify DMRs among the different samples (bin size: $200 \mathrm{bp}$; adjusted $p$-value $<0.1$ for edgeR). The DMRs with a more than twofold change in read counts were classified as hyper- or hypo-methylated regions.

\section{Bisulfite Sequencing of Differentially Methylated Genes}

The differentially methylated gene regions were confirmed by Sanger sequencing. For each target region, primers were designed (Supplementary Table S1). Bisulfite conversions were completed using the EZ DNA Methylation-Gold Kit (Zymo). Briefly, $1.2 \mu \mathrm{g}$ DNA was mixed with the C-T conversion reagent, and then incubated at $98^{\circ} \mathrm{C}$ for $10 \mathrm{~min}, 64^{\circ} \mathrm{C}$ for $2.5 \mathrm{~h}$, and $4^{\circ} \mathrm{C}$ for $30 \mathrm{~min}$. Modified DNA was purified and stored at $-20^{\circ} \mathrm{C}$. We then amplified fragments (approximately $400 \mathrm{bp}$ ). For each PCR, $2.0 \mu \mathrm{l}$ bisulfite-treated DNA was used in a $20-\mu l$ reaction volume. Purified amplicons were cloned into the pEASY-T5 vector (TransGen, Beijing, China) and sequenced. At least 18 clones were sequenced and analyzed

${ }^{1}$ http://broadinstitute.github.io/picard/ for each sample. Methylation rates were expressed as the proportion (\%) per site for each of the three types of cytosines (CG, $\mathrm{CHG}$, and $\mathrm{CHH}$ ). Methylation rates were calculated by dividing the number of non-converted (methylated) cytosines by the total number of cytosines within the assay. The bisulfite sequencing data were analyzed using the Kismeth online tool $^{2}$.

\section{RNA Isolation and Microarray Analysis}

Total RNA was extracted from the leaves of drought-stressed and control DK151 and IR64 plants according to the GeneChip Expression Analysis Technical Manual (Affymetrix). Briefly, total RNA was extracted from frozen leaf tissue using TRIzol reagent and then purified and concentrated using the RNeasy MinElute Cleanup Kit (Qiagen, Germany). CapitalBio Corporation (Beijing) then completed the following steps. Double-stranded cDNA was synthesized from $2 \mu \mathrm{g}$ total RNA. Additionally, biotin-tagged cRNA was generated in an in vitro transcription reaction, which was carried out using the MessageAmp II aRNA Amplification Kit. The resulting cRNA was fragmented (35-200 bases) according to the GeneChip Expression Analysis Technical Manual, and then hybridized to a rice genome array (Affymetrix) containing 48,564 japonica and 1,260 indica sequences. Samples were rotated at $45^{\circ} \mathrm{C}$ for $16 \mathrm{~h}$ in the GeneChip Hybridization Oven 640 (Affymetrix). The chips were then washed and stained in the GeneChip Fluidics Station 450 (Affymetrix), and then scanned using the GeneChip Scanner 3000 (Affymetrix).

Differentially expressed genes (DEGs) (i.e., drought-stressed vs. control samples; genotype vs. genotype) were identified using the two-class unpaired comparison method in the Significant Analysis of Microarray program (criteria: $>2$-fold change and significant $q$-value [false discovery rate-adjusted $p$-value] $<0.05$ based on three independent biological replicates).

To validate the results of transcriptome analysis, a subset of DEGs were verified using qRT-PCR. PCR was conducted according to the methods described by Swarbrick et al. (2008). The sequences of the selected genes were downloaded from the MSU Rice Genome Annotation Project (Kawahara et al., 2013) and used to design primers with Primer $5^{3}$ (Supplementary Table S2). We performed three biological repetitions for each experiment. 49 genes were tested in $50-\mu 1$ reactions using the $\mathrm{SYBR}^{\circledR}$ Green PCR Master Mix kit (Applied Biosystems, Carlsbad, CA, USA) following the manufacturer's protocol.

\section{RESULTS}

\section{Methylated DNA Immunoprecipitation Sequencing Analysis}

The MeDIP-seq libraries were constructed using DNA extracted from drought-stressed DK151 and IR64 samples (i.e., DK151s and IR64s) and control samples (i.e., DK151c and IR64c), and

\footnotetext{
${ }^{2}$ http://katahdin.mssm.edu/kismeth/revpage.pl

${ }^{3}$ http://frodo.wi.mit.edu/
} 
then subjected to high-throughput Illumina sequencing. A total of 21,311,666-21,925,622 raw reads were generated for the four samples. After low-quality data were removed, approximately $85 \%$ of the reads were assessed as clean data and were further analyzed and mapped. An average of approximately 11 million unique mapped reads were obtained for the four samples following a high-quality read alignment against the rice genome (Table 1).

The normalized data ( $\mathrm{reads} / \mathrm{kb}$ ) for the unique mapped reads were further analyzed to characterize the genomic distribution of reads (i.e., upstream of the transcription start site, gene body region, and downstream of the transcription termination site). The MeDIP-seq reads were detected more often in the gene body regions than in the $5^{\prime}$ and $3^{\prime}$ flanking regions (Figure 1), indicating the methylation rate was highest in the gene body regions.

\section{Differential DNA Methylation in DK151 and IR64 under Drought Stress and Control Conditions}

The model-based analysis of MeDIP-seq data detected 27,888 (DK151s), 29,764 (IR64s), 26,189 (DK151c), and 28,515 (IR64c) methylation peaks, indicating there was a greater abundance of DNA methylation peaks in the IR64 line than in the DK151 line (Table 2). The length of the methylation peaks ranged from 200 to $3,500 \mathrm{bp}$, with an average of 2,032 to 2,202 bp in the four samples. Additionally, the total length of the methylation peaks covered approximately $16 \%$ of the whole rice genome (Supplementary Figure S2; Supplementary Table S3). Overall, the peaks were differentially distributed among genomic components, with the distal intergenic regions containing the most methylation peaks (about 35\%), followed by the promoter regions. Within the gene body regions, the methylation peaks were most often detected in the first exon (Table 2).

The methylation peaks in the four samples were compared and summarized in a Venn diagram. More than $70 \%$ of the peaks were common to all four samples, indicating that most of the DNA methylation peaks are relatively stable between the two genotypes under control and drought stress conditions. In contrast, approximately $2-5 \%$ of the peaks were unique to specific samples (Supplementary Figure S2). Examples of the DNA methylation status of the four samples as determined by MeDIP-seq are presented in Figure 2. The DMRs were further analyzed (criteria: $>2$-fold changes and $q$-value $<0.05$ ), and we detected 506 (IR64s vs. IR64c), 92 (DK151s vs. DK151c), 1,190 (DK151c vs. IR64c), and 1,397 (DK151s vs. IR64s) DMRs (Table 3; Figure 3).

\section{Differentially Methylated Regions between DK151 and IR64 under Control and Drought Stress Conditions}

Under normal growth conditions, 500 and 690 DMRs were hyper- and hypo-methylated, respectively, in DK151c plants compared with IR64c plants (Table 3). These DMRs were randomly distributed among the 12 rice chromosomes, and only 125 were located in introgressed regions (Supplementary Table S4), indicating that backcross introgressions resulted in genome-wide DNA methylation changes in the DK151 line. We identified 397 and 556 genes associated with these hyper- and hypo-methylated DMRs, respectively (Supplementary Table S4). Gene ontology (GO) enrichment analysis using agriGO (Du et al., 2010) revealed that the hypo-methylated DMR-associated genes were mainly related to stress response, programmed cell death, and nutrient reservoir activity, while the hyper-methylated DMR-associated genes were mostly related to lyase activity and magnesium ion binding.

We detected 578 and 819 DMRs that were hyper- and hypo-methylated, respectively, in DK151s plants compared with IR64s plants, under drought stress conditions. Most of these DMRs were also observed in the comparison between DK151c and IR64c samples (Figure 3), suggesting that the majority of DNA methylation sites were unaffected by drought stress. These observations are consistent with the analysis of DNA methylation peaks.

\section{Differentially Methylated Regions in DK151 and IR64 Induced by Drought Stress}

We identified 48/44 and 336/170 DMRs that were hyper-/hypomethylated in DK151 and IR64, respectively, under drought stress conditions (relative to the levels in control samples) (Table 3). Overall, there were more hyper-methylated DMRs than hypo-methylated DMRs in both genotypes, implying that hyper-methylation is prevalent in responses to drought stress. Additionally, more DMRs were detected in the drought-sensitive IR64 genotype than in the drought-tolerant DK151 line, with most of these DMRs being genotype-specific (Figure 4). This suggests that DNA methylation changes are highly associated with drought stress responses. Furthermore, the fact that fewer DMRs were observed in the drought-tolerant DK151 than in the drought-sensitive IR64 implies that DNA methylation is more stable in the drought-tolerant genotype.

We identified 48/44 genes associated with the hyper-/hypomethylated DMRs in the DK151s vs. DK151c comparison

TABLE 1 | Summary of MeDIP-seq results for IR64 and DK151 under drought (s) and control (c) conditions.

\begin{tabular}{|c|c|c|c|c|c|}
\hline Sample & Total reads & Mapped reads & Mapped reads in total reads $(\%)$ & Unique mapped reads & Unique mapped reads (\%) \\
\hline IR64c & $21,925,622$ & $18,612,227$ & 84.89 & $11,138,020$ & 50.80 \\
\hline IR64s & $21,311,666$ & $18,255,523$ & 85.58 & $10,960,305$ & 50.20 \\
\hline DK151c & $21,879,742$ & $18,540,441$ & 84.92 & $11,297,601$ & 51.55 \\
\hline DK151s & $21,662,524$ & $18,502,367$ & 85.42 & $11,149,720$ & 51.47 \\
\hline
\end{tabular}




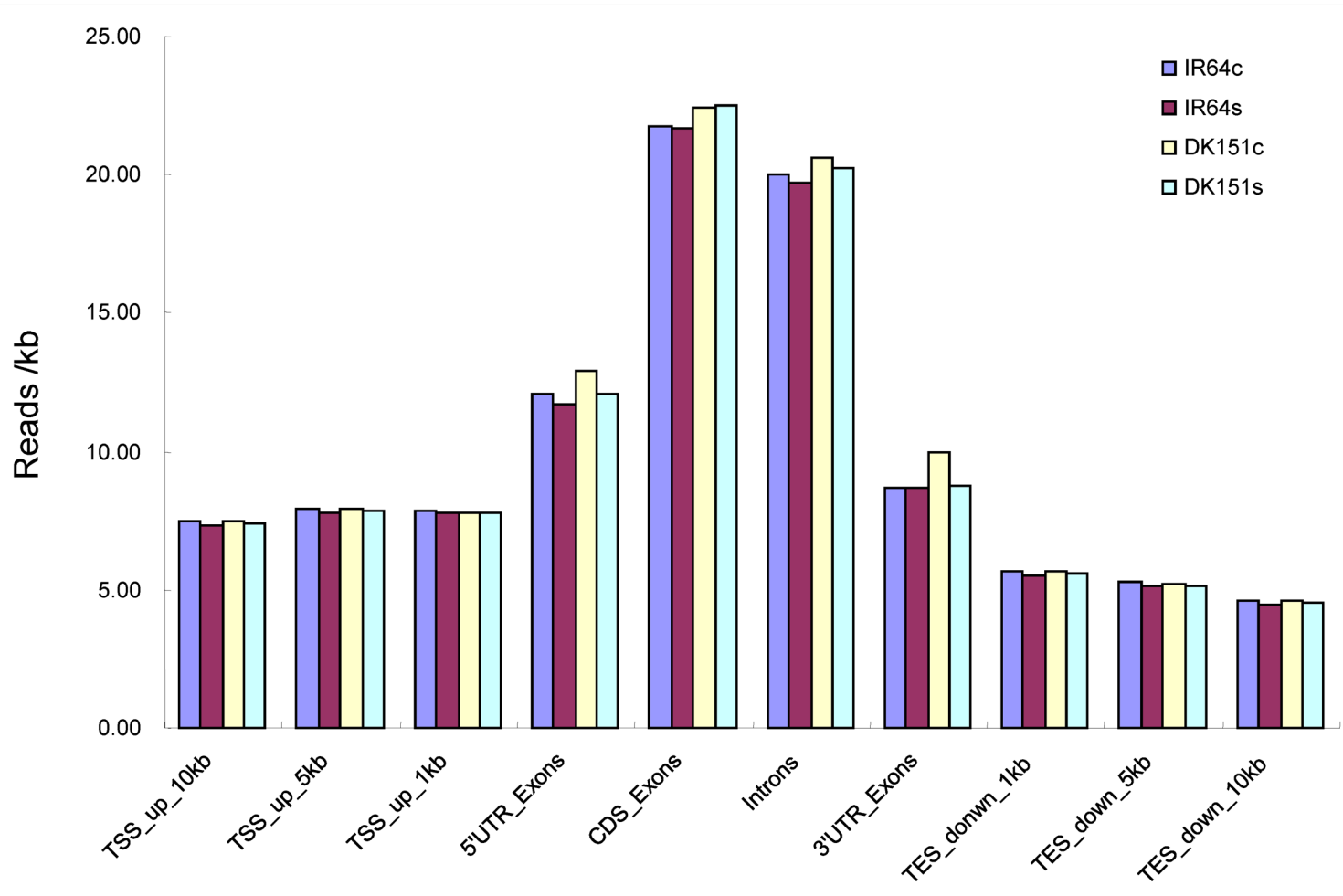

FIGURE 1 | MeDIP-seq read distribution in the genomic regions of DK151 and IR64 under drought stress (s) and control (c) conditions. All uniquely mapped reads upstream of the transcription start site (TSS), 5'UTR, exons, and introns and downstream of the transcription termination site (TES) were calculated and normalized as reads per kilobase (reads/kb).

TABLE 2 | Distribution of methylation peaks in different genomic components.

\begin{tabular}{|c|c|c|c|c|}
\hline Genomic component & IR64c & IR64s & DK151c & DK151s \\
\hline Promoter (2-3 kb) & 4437 (15.56\%) & 4667 (15.68\%) & 3923 (14.98\%) & $4289(15.38 \%)$ \\
\hline Promoter (1-2 kb) & 3949 (13.85\%) & $4259(14.31 \%)$ & 3237 (12.36\%) & $3684(13.21 \%)$ \\
\hline Promoter $(<=1 \mathrm{~kb})$ & $1748(6.13 \%)$ & $1938(6.51 \%)$ & $1587(6.06 \%)$ & $1760(6.31 \%)$ \\
\hline $5^{\prime}$ UTR & $2427(8.51 \%)$ & $2452(8.24 \%)$ & $2514(9.6 \%)$ & $2432(8.72 \%)$ \\
\hline First exon & $2575(9.03 \%)$ & $2536(8.52 \%)$ & 2642 (10.09\%) & 2605 (9.34\%) \\
\hline Other exon & $151(0.53 \%)$ & $173(0.58 \%)$ & $134(0.51 \%)$ & $142(0.51 \%)$ \\
\hline First intron & $63(0.22 \%)$ & $60(0.2 \%)$ & $39(0.15 \%)$ & $53(0.19 \%)$ \\
\hline Other intron & $140(0.49 \%)$ & $149(0.5 \%)$ & $110(0.42 \%)$ & $128(0.46 \%)$ \\
\hline $3^{\prime}$ UTR & $510(1.79 \%)$ & $533(1.79 \%)$ & $490(1.87 \%)$ & $499(1.79 \%)$ \\
\hline Downstream $(<=3 \mathrm{~kb})$ & $2404(8.43 \%)$ & $2464(8.28 \%)$ & $2153(8.22 \%)$ & $2342(8.4 \%)$ \\
\hline Distal intergenic & $10111(35.46 \%)$ & 10533 (35.39\%) & $9360(35.74 \%)$ & 9954 (35.69\%) \\
\hline Total & 28515 (100\%) & 29764 (100\%) & 26189 (100\%) & 27888 (100\%) \\
\hline
\end{tabular}

(Supplementary Table S5). GO analysis revealed that the 44 hypomethylated genes were mostly related to transport, establishment of localization, and nucleotide binding. The genes included those encoding the transcription factors (TFs) TCP, NAC, and WRKY, as well as two cell wall-associated kinase genes (OsWAK2 and OsWAK3), two Osmotin-34 genes, and two $N B-A R C$ genes. The 48 hyper-methylated genes were primarily related to stress responses, transport, and regulation of transcription. The proteins encoded by the hyper-methylated genes included two auxin-responsive factors, the AP2 TF, nuclear transport factor 2 (NTF2), two UDP-glucosyl transferases, and two ARM repeat superfamily proteins. Of the drought-induced DMRs in DK151, only eight (i.e., four hypo- and four hyper-methylated) were localized in introgressed intervals (Supplementary Table S5).

We detected 289/144 genes in the hyper-/hypo-methylated DMRs during the comparison between IR64s and IR64c samples (Supplementary Table S6). GO enrichment analysis results revealed that the 289 hyper-methylated genes were mostly related to programmed cell death, responses to stimuli, transferase activity, and electron carrier activity. In contrast, the 144 hypomethylated genes were mainly associated with responses to stimuli and nutrient reservoir activity. These findings indicate 

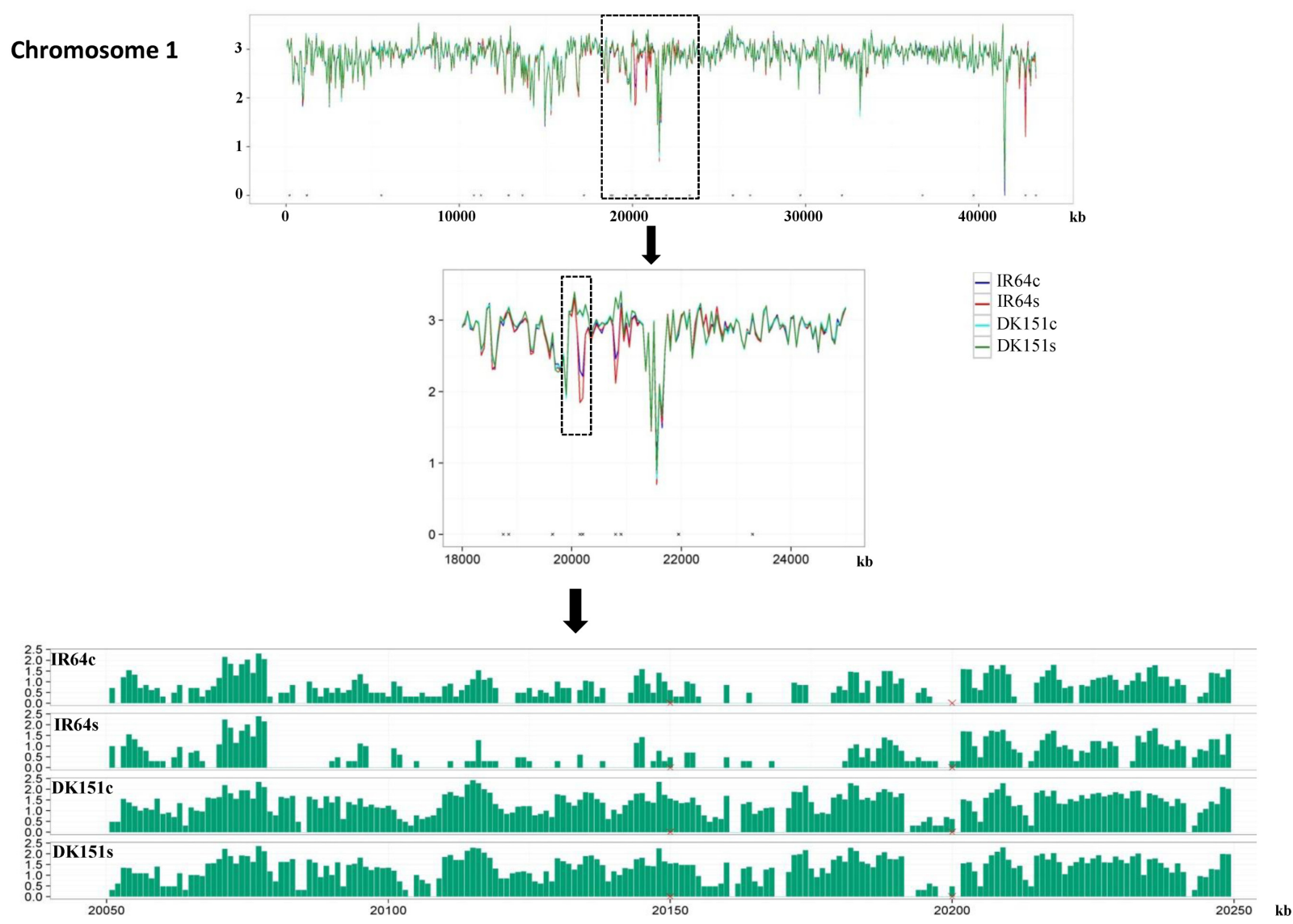

FIGURE 2 | Differential DNA methylation patterns of a region on Chromosome 1 in IR64 and DK151 under drought stress (s) and control (c) conditions.

that drought stress clearly affects the DNA methylation status of these genes in IR64 plants. The GO annotation results suggest that DNA methylation changes affect a diverse range of genes during drought stress responses in the two contrasting genotypes.

Twenty DMR-associated genes were common between the DK151s vs. DK151c and IR64s vs. IR64c comparisons. Most of these genes underwent the same DNA methylation changes in the two genotypes. However, LOC_Os02g29480 (NTF2), LOC_Os06g43590 (vacuolar protein sorting 46.1), and LOC_Os07g10580 (hydrophobic protein LTI6A) exhibited the opposite DNA methylation changes in drought-stressed DK151 and IR64 plants (Supplementary Tables S5 and S6). LOC_Os02g29480 and LOC_Os07g10580 were hypo-methylated (at the $5^{\prime}$ untranslated region) in DK151, but hyper-methylated in IR64. LOC_Os06g43590 was hyper-methylated (at the downstream region) in DK151, but hypo-methylated in IR64. These results indicate these genes are involved in genotypespecific responses to drought conditions.

To confirm the accuracy of the DMR results obtained by MeDIP-seq, several DMR-associated genes underwent bisulfite sequencing, including LOC_Os02g29464 (DNA repair protein RAD50), LOC_Os12g23260 (ureide permease), and LOC_Os12g24020 (glycosyl hydrolase family 9 protein). The bisulfite sequencing data were consistent with the
TABLE 3 | Differentially methylated regions (DMRs) detected between samples.

\begin{tabular}{lccc}
\hline Comparison & $\begin{array}{c}\text { Hyper-methylated } \\
\text { DMRs }\end{array}$ & $\begin{array}{c}\text { Hypo-methylated } \\
\text { DMRs }\end{array}$ & Total \\
\hline IR64s vs. IR64c & 336 & 170 & 506 \\
DK151s vs. DK151c & 48 & 44 & 92 \\
DK151c vs. IR64c & 500 & 690 & 1190 \\
DK151s vs. IR64s & 578 & 819 & 1397 \\
\hline
\end{tabular}

MeDIP-seq profiles. For example, LOC_Os02g23260 was hyper-methylated in IR64 under drought stress conditions, but no DNA methylation changes were detected in this gene in drought-stressed DK151 plants. In contrast, LOC_Os12g23260 and LOC_Os12g24020 were differentially methylated only in DK151 plants exposed to drought stress (Figure 5).

\section{Identification of Differentially Expressed Genes in DK151 and IR64 under Control and Drought Stress Conditions}

To clarify the relationship between DNA methylation and gene expression, we generated transcriptome profiles for DK151 and IR64 using the Affymetrix rice microarray. Under normal growth 


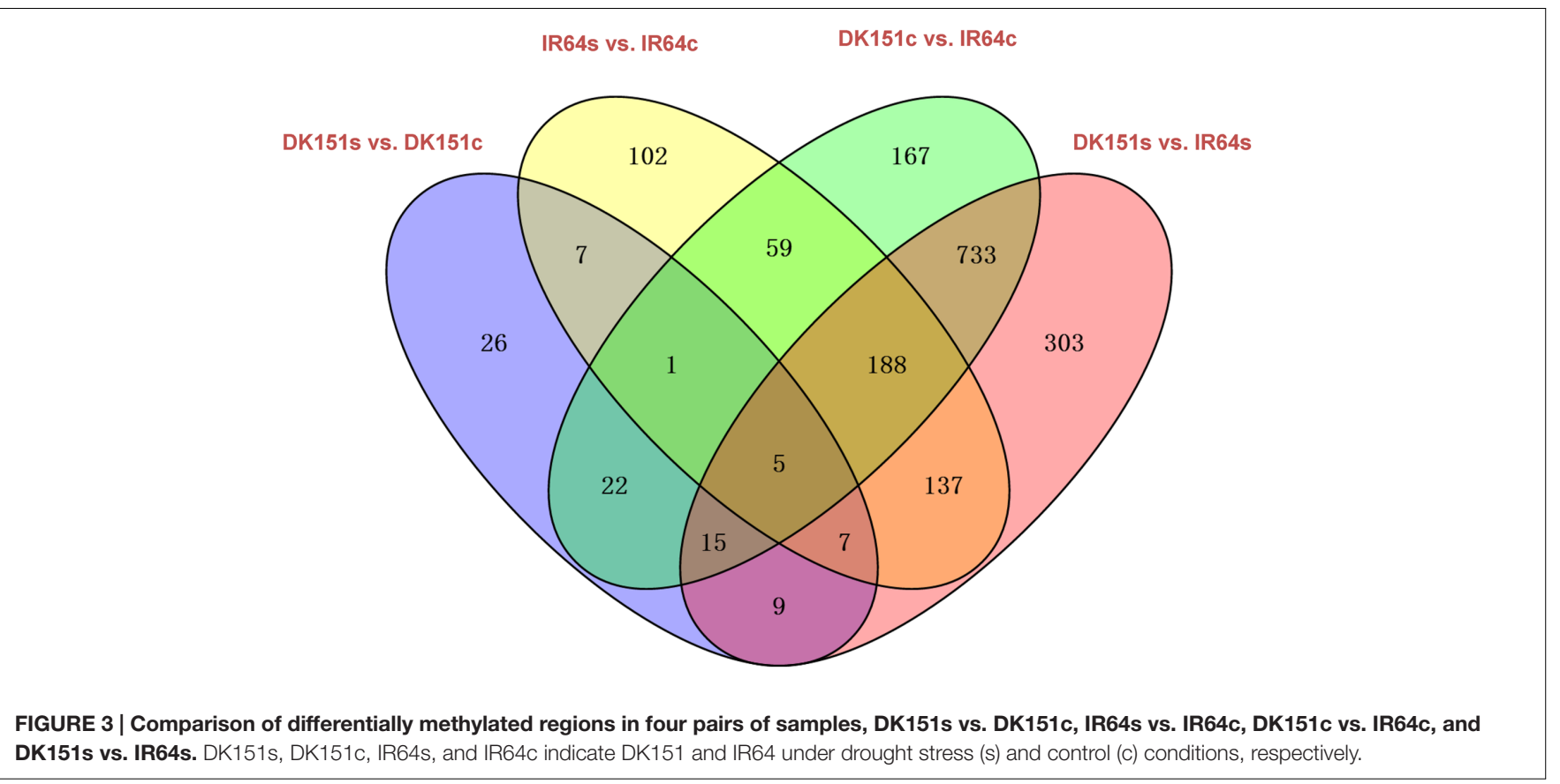

DK151s vs. IR64s. DK151s, DK151c, IR64s, and IR64c indicate DK151 and IR64 under drought stress (s) and control (c) conditions, respectively.

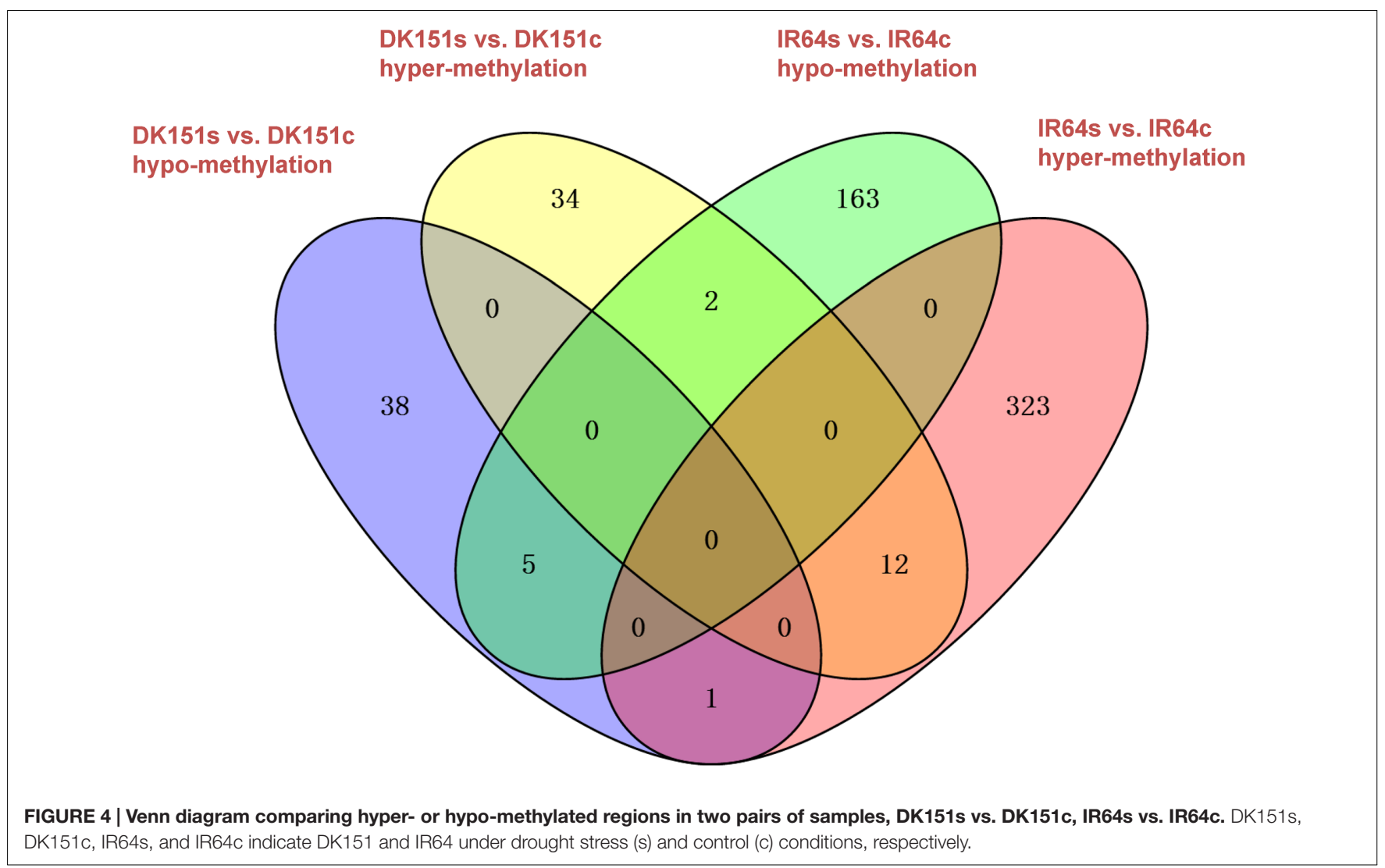

conditions, we detected 3,295 and 2,346 up- and down-regulated genes, respectively, in DK151 plants compared with IR64 plants (Table 4; Supplementary Table S7). This result suggests there are gene expression differences between the two rice genotypes even in the absence of stress. A GO enrichment analysis revealed the DEGs were associated with a diverse range of functional categories, including regulation of transcription, regulation of cellular metabolic process, protein modification, transport, and 
A

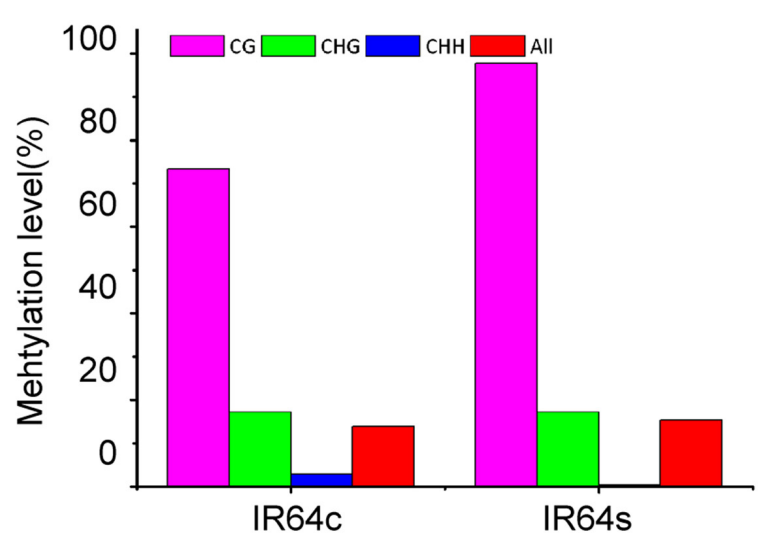

c

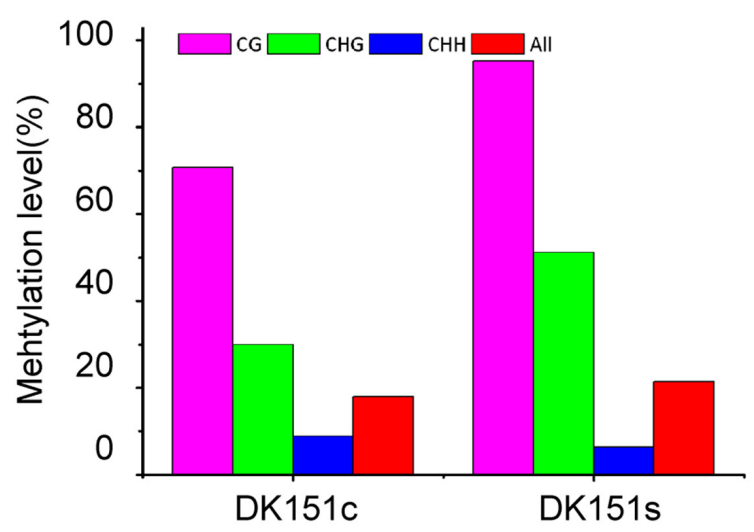

B

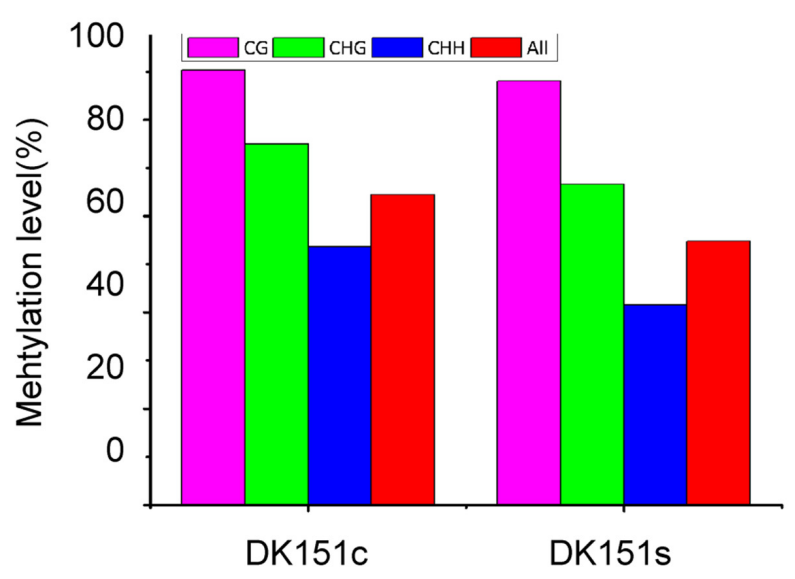

FIGURE 5 | Bisulfite sequencing of three DMR-associated genes. (A) LOC_Os12g23260 (ureide permease); (B) LOC_Os02g29464 (DNA repair protein RAD50); (C) LOC_Os12g24020 (glycosyl hydrolase family 9 protein). The red, green and blue columns in the histograms refer to the collective methylation levels (percentages) of $\mathrm{CG}, \mathrm{CHG}$, and $\mathrm{CH}$, respectively.

TABLE 4 | Differentially expressed genes detected between samples.

\begin{tabular}{lccc}
\hline Comparison & Up-regulated & Down-regulated & Total \\
\hline DK151c vs. IR64C & 3295 & 2346 & 5641 \\
IR64s vs. IR64C & 1840 & 1236 & 3076 \\
DK151s vs. DK151C & 1721 & 1916 & 3637 \\
\hline
\end{tabular}

protein binding (up-regulated genes), as well as translation, small molecular metabolic processes, cellular component biogenesis, and RNA metabolic processes (down-regulated genes).

A comparison between the drought-stressed DK151 and IR64 plants and their respective controls revealed 3,636 (1,721 up-regulated and 1,915 down-regulated) and 3,075 (1,840 upregulated and 1,236 down-regulated) DEGs in DK151 and IR64, respectively (Table 4; Supplementary Tables S8 and S9). Among these, 687 and 550 genes were commonly up- or down-regulated, respectively, in both genotypes (Figure 6). The DK151 upregulated genes were mainly related to carbohydrate metabolic processes, catabolic processes, responses to abiotic stimuli, and transport, while the down-regulated genes were mostly associated with protein modification processes, transcription, regulation of metabolic processes, photosynthesis, and cellular protein metabolic processes. In contrast, the up-regulated IR64 genes were involved in carbohydrate metabolic processes, responses to abiotic stimuli, regulation of gene expression, and localization, while the down-regulated genes were associated with photosynthesis, carbohydrate metabolic processes, oxidationreduction, transport, and membranes. These results indicate that many genes with diverse functions were involved in drought stress responses in DK151 and IR64 plants. Additionally, the genotype-specific GO categories for these DEGs revealed differences in the molecular mechanisms underlying drought stress responses, which were consistent with the contrasting drought tolerance phenotypes.

To validate the transcriptome results, qRT-PCR was used to independently assess expression levels for 49 genes of two genotypes (genes and primer sets are shown in Supplementary Table S2). The strong correlation $\left(R^{2}=0.8503\right)$ between transcript levels and qRT-RCR expression values indicate that there was good agreement between both approaches (Supplementary Figure S3). 


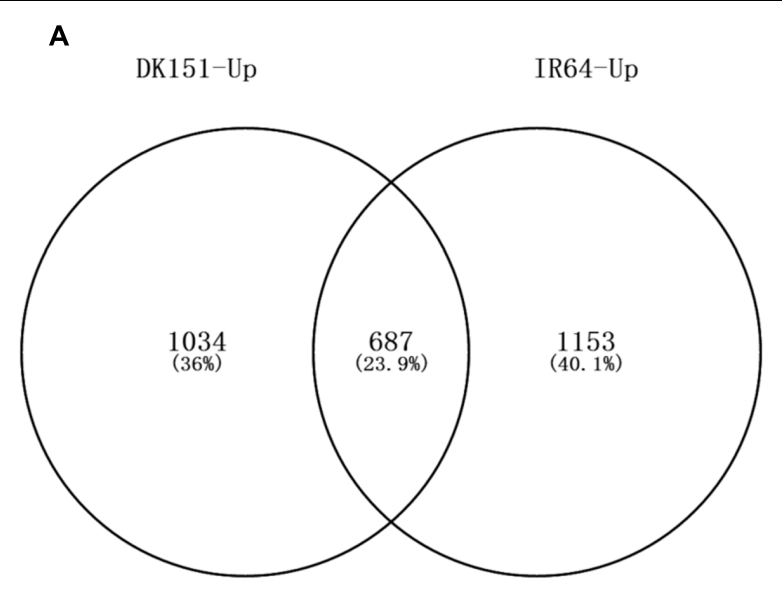

B

DK151-Down IR64-Down

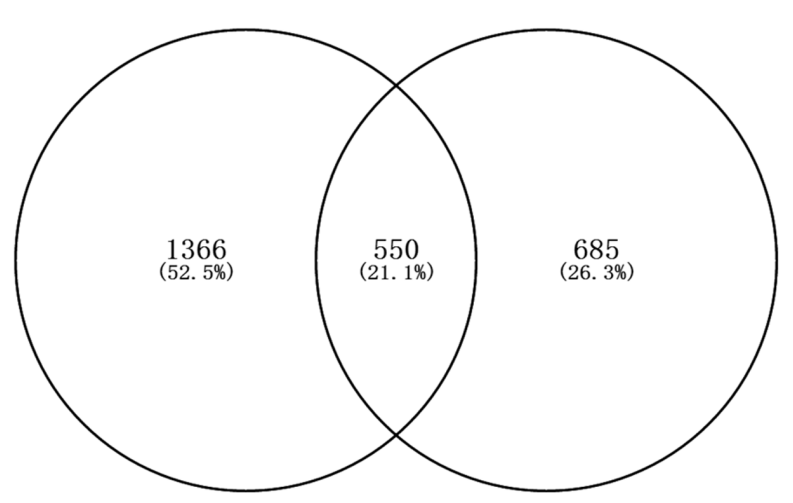

FIGURE 6 | Venn diagram of up-regulated (A) and down-regulated (B) genes in DK151 and IR64 under drought stress compared with respective controls. Differentially expressed genes were identified by twofold changes and significant $q$-values (FDR adjusted $p$-values) less than 0.05 based on three independent biological replicates.

\section{Correlation between Differentially Methylated Regions and Differentially Expressed Genes}

To determine the influence of DNA methylation on gene expression, we first compared the DMRs and DEGs in DK151 with those in IR64 under normal growth conditions. Among the 5,641 DEGs (3,295 up-regulated and 2,346 down-regulated), 103 were located in DMRs, including 41 hyper- and 62 hypo-methylated regions. The hyper-methylated regions were associated with 20 down- and 21 up-regulated genes, whereas the hypo-methylated regions included 21 down- and 41 upregulated genes (Supplementary Table S10). Therefore, there was a negative correlation between the methylation level and transcript abundance for more than half of the DMR-associated DEGs. For example, some genes (e.g., OsPRR9, OsCatalase-2, and OsWRKY11) were more highly expressed in the hypo-methylated regions of DK151 plants than in the IR64 plants. Additionally, the expression levels of genes encoding a cysteine proteinase, histone, $\mathrm{K}^{+}$efflux antiporter-2, a tetratricopeptide repeat-like superfamily protein, and zinc transporter-11 were lower in the hyper-methylated regions of DK151 plants than in the IR64 plants under control conditions.

We also compared the drought-induced DMRs and DEGs from DK151 and IR64 lines. We determined that 12 of 92 DMRs were associated with 12 DEGs in DK151 plants (Supplementary Table S11), while 23 of 506 DMRs were associated with 23 DEGs in IR64 plants (Supplementary Table S12). None of the DMR-associated genes were detected in both genotypes. Genes encoding the LEA protein, NAC TF, Osmotin-34, and leucine-rich receptor-like protein kinase were demethylated, but their expression levels were significantly up-regulated in drought-stressed DK151 plants. Additionally, genes encoding the oxysterol-binding protein and an expressed protein were hyper-methylated, while their expression levels were downregulated in drought-stressed DK151 plants. Genes encoding POZ/BTB-containing G-protein-1, a protein phosphatase 2C family protein, an NB-ARC domain-containing disease resistance protein, trehalose phosphate synthase, a MYB TF, and prefold in subunit 3 were demethylated in IR64 plants exposed to drought conditions, but their expression levels were up-regulated. Three genes encoding proteins with unknown functions were hyper-methylated in drought-stressed IR64 plants, while their expression levels were down-regulated. The remaining DMRassociated genes in the two genotypes exhibited a positive correlation between the methylation level and transcript abundance. Overall, our results indicate that the relationship between DNA methylation and gene expression is complex.

\section{DISCUSSION}

\section{Methylated DNA Immunoprecipitation Sequencing Analysis of Two Contrasting Drought-Tolerant Genotypes}

DNA methylation has been widely implicated in physiological and developmental processes in plants. With the development of next-generation sequencing technology, several genomewide DNA methylation profiling platforms are now viable options, including bisulfite sequencing and MeDIP-seq. Bisulfite sequencing is still considered the gold standard for profiling DNA methylation at a single base pair resolution (Li et al., 2010; Taiwo et al., 2012; Crampton et al., 2016). However, MeDIPseq is a high-throughput and more affordable method that may be appropriate for routine use, even though the coverage and resolution are lower. In this study, we used MeDIP-seq to profile the DNA methylation status of a drought-tolerant introgression rice line (DK151) and its recurrent parent (IR64) under drought and control conditions. The MeDIP-seq data indicated that DNA methylation is more common in the gene body regions than in the other gene regions. This result is consistent with those of a previous study (Zhang et al., 2006). Analyses of methylation peaks revealed that more than one-third of the peaks are located 
in intergenic regions, which is similar to the methylation peak distribution pattern in the Arabidopsis thaliana (Yong-Villalobos et al., 2015) and poplar genomes (Vining et al., 2012).

\section{Introgression and DNA Methylation Changes}

Alien introgressions may result in genome-wide DNA methylation changes in animals (Müller et al., 2001) and plants (Liu et al., 2004; Dong et al., 2006). In this study, more DMRs were detected in the introgression line DK151 than in its recurrent parent IR64 under normal growth conditions. These DMRs were evenly distributed among 12 chromosomes. Only 125 of 1,190 DMRs overlapped with introgressed regions, indicating that the introgression of donor genomic DNA induces genome-wide DNA methylation changes. The extensive epigenetic effects of alien introgressions may result from a general epigenetic disturbance, the introduction of exogenous methylation-modifying factors from the donor, or genomic rearrangements (Müller et al., 2001; Riddle and Richards, 2002; Dong et al., 2006). However, the molecular mechanisms regulating these considerable DNA methylation changes require further study.

There is evidence indicating that altered DNA methylation induced by alien chromosomal segments may have contributed to the development of abiotic stress tolerance (Wang M. et al., 2014). More interestingly, GO enrichment analyses revealed that most of the DMR-associated genes in DK151 are related to stress responses, programmed cell death, nutrient reservoir activity, lyase activity, and magnesium ion binding. This observation implies that introgression-induced changes to the DNA methylation status of these genes may be correlated with the constitutive drought tolerance of DK151 plants.

\section{Functions of DNA Methylation in Drought Stress Tolerance}

The extent and pattern of DNA methylation differ among diverse rice genotypes with various stress tolerance phenotypes (Zheng et al., 2013; Garg et al., 2015). There is also evidence that genome-wide DNA methylation changes are involved in abiotic stress responses in plants (Eichten and Springer, 2015). In this study, numerous DMRs were detected in both drought-stressed genotypes, indicating that drought-induced DNA methylation changes have a considerable impact on drought stress responses in rice plants. There were fewer drought-induced DMRs detected in DK151 plants than in IR64 plants, which further confirms the results of a previous study that concluded that the methylome is more stable in a drought-tolerant genotype than in a droughtsensitive genotype during exposures to drought conditions (Zheng et al., 2013).

Gene ontology analyses of DMR-associated genes in DK151 plants under drought conditions revealed that the hypo- or hyper-methylated genes are mainly related to transport, stress responses, and transcription regulation. Additionally, several TF genes, including those encoding WRKY, TCP, NAC, ARF, and AP2, were uniquely differentially methylated in DK151 plants under drought stress. This implies that drought-induced
DNA methylation changes may regulate the expression of genes associated with drought stress responses by affecting TF genes. Our results are consistent with those of a previous study that revealed stress-induced DNA methylation changes of TF genes are repressed or activated in soybean plants under salt stress conditions (Song et al., 2012). Two cell wall-associated kinase genes (OsWAK2 and OsWAK3) and two Osmotin-34 genes were only hypo-methylated in DK151 under drought conditions. These genes influence stress responses (Zhang et al., 2005; de Oliveira et al., 2014), and the decreased DNA methylation may positively affect the drought tolerance of DK151 plants.

The genes encoding NTF2, vacuolar protein sorting 46.1, and hydrophobic protein LTI6A exhibited contrasting DNA methylation patterns in DK151 and IR64 plants under drought conditions. The NTF2 protein is essential for nuclear trafficking, and is involved in importing proteins into the nucleus (Zhao et al., 2006). The expression of NTF2 was up-regulated by drought independently of abscisic acid (Hu et al., 2011). Vacuolar protein sorting 46.1 is an endosomal sorting complex required for transport-related protein, and it affects intracellular protein trafficking (Spitzer et al., 2009). Hydrophobic protein LTI6A is a transmembrane protein that may influence transport (Rudd et al., 1998). Differences in the changes to the DNA methylation of these three genes imply that the transport of biomacromolecules in DK151 and IR64 plants under drought conditions is regulated by epigenetic remodeling. These observations suggest that DNA methylation changes influence drought stress responses by epigenetically regulating genes with diverse functions.

\section{DNA Methylation has Diverse Effects on Gene Expression}

The regulatory effects of DNA methylation on plant gene expression have been widely studied. Methylation of the promoter region generally represses gene expression while the methylation of the gene body has the opposite effect (Zilberman et al., 2007; Li et al., 2012). In this study, we compared the methylome and transcriptome of two rice genotypes with contrasting drought tolerance phenotypes. Our results revealed that the relationship between DNA methylation status and gene expression level is more complex than expected. First, we detected 1,190 DMRs and 5,641 DEGs between DK151 and IR64 under normal growth conditions. Only 103 DEGs were located in 41 hyper- and 62 hypo-methylated DMRs, indicating that a small proportion of the DMR-associated genes exhibit a significantly different expression level between the two genotypes. The same trend was observed for the drought-induced DMRs and DEGs for both genotypes, with only a few DMR-associated genes differentially expressed between the drought-stressed and control plants. These results are consistent with those of several previous studies that revealed that in most cases, differences in gene expression levels are not correlated with differences in DNA methylation (Garg et al., 2015; Secco et al., 2015; Wang et al., 2015). The effects of DNA methylation on gene expression may be directly or indirectly mediated through common or unique transcriptional pathways. 
Second, we observed positive and negative correlations between DNA methylation and gene expression. A negative correlation between methylation level and transcript abundance was detected for more than half of the DMR-associated genes, which is in agreement with the findings of several previous studies (Zilberman et al., 2007; Li et al., 2012; Vining et al., 2012; Hu et al., 2015). However, we identified many DK151 and IR64 genes for which there was a positive relationship between DNA methylation and transcript abundance under control and drought stress conditions. The DMRs in different gene regions have diverse effects on gene expression (Liang et al., 2011; Dubin et al., 2015), indicating there is no direct linear relationship between the methylome and transcriptome. Furthermore, the effects of DNA methylation on plant gene expression were observed to be tissue/organ-specific (GutierrezArcelus et al., 2015) and dependent on the developmental stage (Wang et al., 2015; Yong-Villalobos et al., 2015). Specifically, the DNA methylation changes in drought-stressed plants occurred primarily in genes that encoded proteins with unique functions. This is consistent with the results of earlier studies (Zilberman et al., 2007; Wang J. et al., 2014; Colicchio et al., 2015). Therefore, DNA methylation has diverse effects on gene expression, and the relationship between methylation status and expression is more complicated than expected.

\section{CONCLUSION}

We detected genome-wide differences in DNA methylation between the drought-tolerant introgression line DK151 and its drought-sensitive recurrent parent IR64 under drought and control conditions. The introgression induced global DNA methylation changes in DK151 are implicated to its constitutive

\section{REFERENCES}

Bender, J. (2004). DNA methylation and epigenetics. Annu. Rev. Plant Biol. 55, 41-68. doi: 10.1146/annurev.arplant.55.031903.141641

Boyko, A., and Kovalchuk, I. (2008). Epigenetic control of plant stress response. Environ. Mol. Mutagen. 49, 61-72. doi: 10.1002/em.20347

Cai, Q., and Chinnappa, C. C. (1999). Cytosine methylation levels in the genome of Stellaria longipes. J. Biosci. 24, 27-33. doi: 10.1007/BF02941103

Colicchio, J. M., Miura, F., Kelly, J. K., Ito, T., and Hileman, L. C. (2015). DNA methylation and gene expression in Mimulus guttatus. BMC Genomics 16:507. doi: 10.1186/s12864-015-1668-0

Crampton, M., Sripathi, V. R., Hossain, K., and Kalavacharla, V. (2016). Analyses of methylomesderived from Meso-American common bean (Phaseolus vulgaris L.) using MeDIP-seq and whole genome sodium bisulfite-sequencing. Front. Plant Sci. 7:447. doi: 10.3389/fpls.2016.00447

Datta, S. K., Malabuyac, J. A., and Aragon, E. L. (1988). A field screening technique for evaluating rice germplasm for drought tolerance during the vegetative stage. Field Crops Res. 19, 123-134. doi: 10.1016/0378-4290(88)90050-0

de Oliveira, L. F., Christoff, A. P., de Lima, J. C., de Ross, B. C., Sachetto-Martins, G., Margis-Pinheiro, M., et al. (2014). The Wall-associated Kinase gene family in rice genomes. Plant Sci. 229, 181-192. doi: 10.1016/j.plantsci.2014.09.007

Degenkolbe, T., Do, P. T., Zuther, E., Repsilber, D., Walther, D., Hincha, D. K., et al. (2009). Expression profiling of rice cultivars differing in their tolerance to long-term drought stress. Plant Mol. Biol. 69, 133-153. doi: 10.1007/s11103008-9412-7 drought tolerance. And drought stress induced differential DNA methylation alterations in two genotypes, the DMR-associated genes are involved in genotype-specific drought responses. A comparison of the methylome and transcriptome revealed that the relationship between DNA methylation and gene expression is complex. Some genes that were differentially methylated exhibited significantly altered gene expression, implying they may be involved in the molecular regulation of drought stress responses.

\section{AUTHOR CONTRIBUTIONS}

WW and QQ performed the DNA methylation and transcriptome analyses, FS, YW and DX performed sampling and bisulfate sequencing analyses, $\mathrm{ZL}$ and $\mathrm{BF}$ interpreted data and wrote the manuscript.

\section{ACKNOWLEDGMENTS}

This work was supported by grants from National Natural Science Foundation of China (31271694, 31571631, 31501291, and 31261140369), The National Key Research and Development Program of China (2016YFD0100904), the Shenzhen Peacock Plan (20130415095710361) and the CAAS Innovative Team Award.

\section{SUPPLEMENTARY MATERIAL}

The Supplementary Material for this article can be found online at: http://journal.frontiersin.org/article/10.3389/fpls.2016.01675/ full\#supplementary-material

Dong, Z. Y., Wang, Y. M., Zhang, Z. J., Shen, Y., Lin, X. Y., Ou, X. F., et al. (2006). Extent and pattern of DNA methylation alteration in rice lines derived from introgressive hybridization of rice and Zizanialatifolia Griseb. Theor. Appl. Genet. 113, 196-205. doi: 10.1007/s00122-006-0286-2

Du, Z., Zhou, X., Ling, Y., Zhang, Z., and Su, Z. (2010). agriGO: a GO analysis toolkit for the agricultural community. Nucleic Acids Res. 38, W64-W70. doi: 10.1093/nar/gkq310

Dubin, M. J., Zhang, P., Meng, D., Remigereau, M. S., Osborne, E. J., Paolo Casale, F., et al. (2015). DNA methylation in Arabidopsis has a genetic basis and shows evidence of local adaptation. Elife 4:e05255. doi: 10.7554/eLife.05255

Eichten, S. R., and Springer, N. M. (2015). Minimal evidence for consistent changes in maize DNA methylation patterns following environmental stress. Front. Plant Sci. 6:308. doi: 10.3389/fpls.2015.00308

Farooq, M., Wahid, A., Kobayashi, N., Fujita, D., and Basra, S. M. A. (2009). Plant drought stress: effects, mechanisms and management. Agron. Sustain. Dev. 29, 185-212. doi: 10.1051/agro:2008021

Finnegan, E. J., Genger, R. K., Peacock, W. J., and Dennis, E. S. (1998). DNA methylation in plants. Annu. Rev. Plant Physiol. Plant Mol. Biol. 49, 223-247. doi: 10.1146/annurev.arplant.49.1.223

Garg, R., Chevala, V. N., Shankar, R., and Jain, M. (2015). Divergent DNA methylation patterns associated with gene expression in rice cultivars with contrasting drought and salinity stress response. Sci. Rep. 5:14922. doi: 10.1038/srep14922

Gutierrez-Arcelus, M., Ongen, H., Lappalainen, T., Montgomery, S. B., Buil, A., Yurovsky, A., et al. (2015). Tissue-specific effects of genetic and epigenetic 
variation on gene regulation and splicing. PLoS Genet. 11:e1004958. doi: 10.1371/journal.pgen.1004958

Hu, J., Chen, X., Zhang, H., and Ding, Y. (2015). Genome-wide analysis of DNA methylation in photoperiod- and thermo-sensitive male sterile rice Peiai 64S. BMC Genomics 16:102. doi: 10.1186/s12864-015-1317-7

Hu, X. L., Lu, M. H., Li, C. H., Liu, T. X., Wang, W., Wu, J. Y., et al. (2011). Differential expression of proteins in maize roots in response to abscisic acid and drought. Acta Physiol. Plant. 33, 2437-2446. doi: 10.3732/ajb. 90.4.526

Huang, L. Y., Zhang, F., Zhang, F., Wang, W. S., Zhou, Y. L., Fu, B. Y., et al. (2014). Comparative transcriptome sequencing of tolerant rice introgression line and its parents in response to drought stress. BMC Genomics 15:1026. doi: 10.1186/1471-2164-15-1026

Karan, R., DeLeon, T., Biradar, H., and Subudhi, P. K. (2012). Salt stress induced variation in DNA methylation pattern and its influence on gene expression in contrasting rice genotypes. PLOS ONE 7:e4020. doi: 10.1371/journal.pone.0040203

Kawahara, Y., de la Bastide, M., Hamilton, J. P., Kanamori, H., McCombie, W. R., Ouyang, S., et al. (2013). Improvement of the Oryza sativa Nipponbare reference genome using next generation sequence and optical map data. Rice 6:4. doi: 10.1186/1939-8433-6-4

Li, N., Ye, M., Li, Y., Yan, Z., Butcher, L. M., Sun, J., et al. (2010). Whole genome DNA methylation analysis based on high throughput sequencing technology. Methods 52, 203-212. doi: 10.1016/j.ymeth.2010. 04.009

Li, X., Zhu, J. D., Hu, F. Y., Ge, S., Ye, M. Z., Xiang, H., et al. (2012). Singlebase resolution maps of cultivated and wild rice methylomes and regulatory roles of DNA methylation in plant gene expression. BMC Genomics 13:300. doi: 10.1186/1471-2164-13-300

Liang, P., Song, F., Ghosh, S., Morien, E., Qin, M., Mahmood, S., et al. (2011). Genome-wide survey reveals dynamic widespread tissue-specific changes in DNA methylation during development. BMC Genomics 12:231. doi: 10.1186/1471-2164-12-231

Lienhard, M., Grimm, C., Morkel, M., Herwig, R., and Chavez, L. (2014). MEDIPS: genome-wide differential coverage analysis of sequencing data derived from DNA enrichment experiments. Bioinformatics 30, 284-286. doi: 10.1093/bioinformatics/btt650

Liu, Z., Wang, Y., Shen, Y., Guo, W., Hao, S., and Liu, B. (2004). Extensive alterations in DNA methylation and transcription in rice caused by introgression from Zizanialatifolia. Plant Mol. Biol. 54, 571-582. doi: 10.1023/B:PLAN.0000038270.48326.7a

Lu, Y. C., Feng, S. J., Zhang, J. J., Luo, F., Zhang, S., and Yang, H. (2016). Genomewide identification of DNA methylation provides insights into the association of gene expression in rice exposed to pesticide atrazine. Sci. Rep. 6:18985. doi: 10.1038/srep 18985

Meyer, P. (2015). Epigenetic variation and environmental change. J. Exp. Bot. 66, 3541-3548. doi: 10.1093/jxb/eru502

Müller, K., Heller, H., and Doerfler, W. (2001). Foreign DNA integration. Genomewide perturbations of methylation and transcription in the recipient genomes. J. Biol. Chem. 276, 14271-14278.

Riddle, N. C., and Richards, E. J. (2002). The control of natural variation in cytosine methylation in Arabidopsis. Genetics 162, 355-363.

Rudd, K. E., Humphery-Smith, I., Wasinger, V. C., and Bairoch, A. (1998). Low molecular weight proteins: a challenge for postgenomic research. Electrophoresis 19, 536-544. doi: 10.1002/elps.1150 190413

Secco, D., Wang, C., Shou, H., Schultz, M. D., Chiarenza, S., Nussaume, L., et al. (2015). Stress induced gene expression drives transient DNA methylation changes at adjacent repetitive elements. Elife 4:e09343. doi: 10.7554/eLife. 09343

Song, Y., Ji, D., Li, S., Wang, P., Li, Q., and Xiang, F. (2012). The dynamic changes of DNA methylation and histone modifications of salt responsive transcription factor genes in soybean. PLoS ONE 7:e41274. doi: 10.1371/journal.pone.0041274

Spitzer, C., Reyes, F. C., Buono, R., Sliwinski, M. K., Haas, T. J., and Otegui, M. S. (2009). The ESCRT-related CHMP1A and B proteins mediate multivesicular body sorting of auxin carriers in Arabidopsis and are required for plant development. Plant Cell 21, 749-766. doi: 10.1105/tpc.108.064865
Swarbrick, P. J., Huang, K., Liu, G., Slate, J., Press, M. C., and Scholes, J. D. (2008). Global patterns of gene expression in rice varieties undergoing a susceptible or resistant interaction with the parasitic Striga hermonthica. New Phytol. 179, 515-529. doi: 10.1111/j.1469-8137.2008.02484.x

Taiwo, O., Wilson, G. A., Morris, T., Seisenberger, S., Reik, W., Pearce, D., et al. (2012). Methylome analysis using MeDIP-seq with low DNA concentrations. Nat. Protoc. 7, 617-636. doi: 10.1038/nprot.2012.012

Tang, X. M., Tao, X., Wang, Y., Ma, D. W., Li, D., Yang, H., et al. (2014). Analysis of DNA methylation of perennial ryegrass under drought using the methylation-sensitive amplification polymorphism (MSAP) technique. Mol. Genet. Genomics 289, 1075-1084. doi: 10.1007/s00438-014-0869-6

Todaka, D., Shinozaki, K., and Yamaguchi-Shinozaki, K. (2015). Recent advances in the dissection of drought-stress regulatory networks and strategies for development of drought-tolerant transgenic rice plants. Front. Plant Sci. 6:84. doi: 10.3389/fpls.2015.00084

Vining, K. J., Pomraning, K. R., Wilhelm, L. J., Priest, H. D., Pellegrini, M., Mockler, T. C., et al. (2012). Dynamic DNA cytosine methylation in the Populus trichocarpa genome: tissue-level variation and relationship to gene expression. BMC Genomics 13:27. doi: 10.1186/1471-2164-13-27

Wang, J., Marowsky, N. C., and Fan, C. (2014). Divergence of gene body DNA methylation and evolution of plant duplicate genes. PLOS ONE 9:e110357. doi: 10.1371/journal.pone.0110357

Wang, M., Qin, L. M., Xie, C., Li, W., Yuan, J. R., Kong, L. N., et al. (2014). Induced and constitutive DNA methylation in a salinity-tolerant whet introgression line. Plant Cell Physiol. 55, 1354-1365. doi: 10.1093/pcp/pcu059

Wang, P., Xia, H., Zhang, Y., Zhao, S., Zhao, C., Hou, L., et al. (2015). Genome-wide high-resolution mapping of DNA methylation identifies epigenetic variation across embryo and endosperm in Maize (Zea may). BMC Genomics 16:21. doi: 10.1186/s12864-014-1204-7

Wang, W. S., Pan, Y. J., Zhao, X. Q., Dwivedi, D., Zhu, L. H., Ali, J., et al. (2011a). Drought-induced site-specific DNA methylation and itsassociation with drought tolerance in rice (Oryza sativa L.). J. Exp. Bot. 62, 1951-1960. doi: $10.1093 / \mathrm{jxb} / \mathrm{erq} 391$

Wang, W. S., Zhao, X. Q., Pan, Y. J., Zhu, L. H., Fu, B. Y., and Li, Z. (2011b). DNA methylation changes detected by methylation-sensitive amplified polymorphism in two contrasting rice genotypes under salt stress. J. Genet. Genomics 38, 419-424. doi: 10.1016/j.jgg.2011.07.006

Xing, M. Q., Zhang, Y. J., Zhou, S. R., Hu, W. Y., Wu, X. T., Ye, Y. J., et al. (2015). Global analysis reveals the crucial roles of DNA methylation during rice seed development. Plant Physiol. 168, 1417-1432. doi: 10.1104/pp.15.00414

Xiong, L. Z., Xu, C. G., Maroof, M. A. S., and Zhang, Q. F. (1999). Patterns of cytosine methylation in elite rice hybrid and its parental lines, detected by a methylation sensitive amplification polymorphism technique. Mol. Gen. Genet. 261, 439-446. doi: 10.1007/s004380050986

Yong-Villalobos, L., González-Morales, S. I., Wrobel, K., Gutiérrez-Alanis, D., Cervantes-Peréz, S. A., Hayano-Kanashiro, C., et al. (2015). Methylome analysis reveals an important role for epigenetic changes in the regulation of the Arabidopsis response to phosphate starvation. Proc. Natl. Acad. Sci. U.S.A. 112, E7293-E7302. doi: 10.1073/pnas.1522301112

Zhang, S., Chen, C., Li, L., Meng, L., Singh, J., Jiang, N., et al. (2005). Evolutionary expansion, gene structure, and expression of the rice wall-associated kinase gene family. Plant Physiol. 139, 1107-1124. doi: 10.1104/pp.105.069005

Zhang, X., Yazaki, J., Sundaresan, A., Cokus, S., Chan, S. W., Chen, H., et al. (2006). Genome-wide high-resolution mapping and functional analysis of DNA methylation in Arabidopsis. Cell 126, 1189-1201. doi: 10.1016/j.cell.2006.08.003

Zhang, Y., Liu, T., Meyer, C. A., Eeckhoute, J., Johnson, D. S., Bernstein, B. E., et al. (2008). Model-based analysis of ChIP-Seq (MACS). Genome Biol. 9:R137. doi: 10.1186/gb-2008-9-9-r137

Zhao, Q., Leung, S., Corbett, A. H., and Meier, I. (2006). Identification and characterization of the Arabidopsis orthologs of nuclear transport factor 2, the nuclear import factor of ran. Plant Physiol. 140, 869-878. doi: 10.1104/pp.105.075499

Zheng, X., Chen, L., Li, M., Lou, Q., Xia, H., Wang, P., et al. (2013). Transgenerational variations in DNA methylation induced by drought stress in two rice varieties with distinguished difference to drought resistance. PLoS ONE 8:e80253. doi: 10.1371/journal.pone.0080253

Zilberman, D., Gehring, M., Tran, R. K., Ballinger, T., and Henikoff, S. (2007). Genome-wide analysis of Arabidopsis thaliana DNA methylation uncovers an 
interdependence between methylation and transcription. Nat. Genet. 39, 61-69. doi: $10.1038 / \mathrm{ng} 1929$

Conflict of Interest Statement: The authors declare that the research was conducted in the absence of any commercial or financial relationships that could be construed as a potential conflict of interest.
Copyright $\odot 2016$ Wang, Qin, Sun, Wang, Xu, Li and Fu. This is an open-access article distributed under the terms of the Creative Commons Attribution License (CC BY). The use, distribution or reproduction in other forums is permitted, provided the original author(s) or licensor are credited and that the original publication in this journal is cited, in accordance with accepted academic practice. No use, distribution or reproduction is permitted which does not comply with these terms. 